\title{
Activités antimicrobiennes des plantes utilisées comme bâtonnets frotte-dents (cure-dents) par les Peul de la commune de Tessékéré (Ferlo Nord, Sénégal)
}

\author{
Bétémondji Désiré DIATTA ${ }^{1 *}$, Emeline HOUËL ${ }^{2}$, Mathieu GUEYE$^{1}$, Ousmane NIASS $^{3}$ et \\ Gilles BOETSCH${ }^{4}$
}

\author{
${ }^{1}$ Laboratoire de Botanique, Département de Botanique et Géologie, IFAN Cheikh. A. Diop/UCAD BP 206 \\ Dakar ; UMI 3189 « Environnement, Santé et Société », Faculté de Médecine Pharmacie Odontostomatologie \\ $U C A D$. \\ ${ }^{2}$ CNRS, UMR EcoFoG, AgroParisTech, Cirad, INRA, Université des Antilles, Université de Guyane, 97300 \\ Cayenne, France. \\ ${ }^{3}$ Laboratoire de chimie analytique Faculté de Médecine Pharmacie Odontostomatologie à l'université Cheikh \\ Anta Diop de Dakar, Sénégal. \\ ${ }^{5}$ CNRS, UMI 3189 Environnement, santé société, faculté de médecine secteur Nord, Marseille, France et \\ OHMi Téssékéré (CNRS, INEE - UCAD). \\ *Auteur correspondant ; E-mail: betemondjidesire@hotmail.fr ; Tel: +221777963332
}

\section{RESUME}

Le bâtonnet frotte-dent est le matériel d'hygiène dentaire le plus communément utilisé au Ferlo pour prévenir les pathologies bucco-dentaires dentaires et garder une belle dentition. Afin d'évaluer l'activité biologique des plantes utilisées comme bâtonnets frotte-dents dans la commune de Tessékéré (Nord Sénégal), une enquête ethnobotanique au moyen d'un guide d'entretien semi-directif a été effectuée pour recenser les plantes utilisées. Parmi les espèces citées, treize, réparties dans huit familles ont été retenues sur la base de l'importance de leur Indice de Fidélité (IF). Des extraits aqueux ont été réalisés à partir des tiges en vue de l'évaluation leurs activités antimicrobiennes. Des tests de micro dilution ont permis d'évaluer la concentration inhibitrice minimale (CIM) des extraits des plantes sélectionnées sur trois souches bactériennes pathogènes bucco-dentaires (Streptoccocus mutans, Porphyromonas gingivalis et Lactobacillus acidophilus) et deux souches de Candida albicans, levures opportunistes. Les extraits d'Anogeissus leiocarpa, de Sclerocarya birrea et d'Acacia seyal se sont révélés actifs. Anogeissus leiocarpa, plante la plus active, présente un effet significatif contre les deux souches de $C$. albicans et deux souches bactériennes (L. acidophilus et $P$. gingivalis) sur les trois testées, avec des valeurs de CIM comprises entre 16 et $512 \mu \mathrm{g} / \mathrm{ml}$. L'extrait de $S$. birrea a montré une activité modérée contre une des deux souches de C. albicans, de même que celui d'Acacia seyal vis-à-vis de Streptoccocus mutans. Ces résultats mettent en évidence l'intérêt de ces trois espèces ligneuses dans le cadre de l'hygiène bucco-dentaire comme bâtonnets frotte-dents.

(C) 2019 International Formulae Group. All rights reserved.

Mots clés: Bâtonnets frotte-dents, Activités antimicrobiennes, Peul, Tessékéré, Ferlo Nord, Sénégal.

\section{Antimicrobial activities of plants used as toothbrush (toothpicks) by Fulani of Tessékéré commune (North Ferlo, Senegal)}

\begin{abstract}
The toothbrush stick is the most commonly used dental hygiene material at Ferlo to prevent dental pathologies and keep a beautiful dentition. In order to evaluate the biological activity of the plants used as
\end{abstract}


rubbing sticks in the municipality of Tessékéré (North Senegal), an ethnobotanical survey using a semidirective interview guide was conducted to identify the plants used. Among the species mentioned, thirteen, divided into eight families were selected on the basis of the importance of their Fidelity Index (FI). Aqueous extracts were made from the stems for evaluation of associated antimicrobial activities. Micro dilution tests were used to evaluate the minimum inhibitory concentration (MIC) of selected plant extracts on three bacterial oral pathogenic strains (Streptoccocus mutans, Porphyromonas gingivalis and Lactobacillus acidophilus) and two strains of Candida albicans (opportunistic yeasts). The extracts of Anogeissus leiocarpa, Sclerocarya birrea and Acacia seyal proved to be active. A. leiocarpa, the most active plant, has a significant effect against both strains of $C$. albicans and two bacterial strains (L. acidophilus and P. gingivalis) on the three tested, with MIC values between 16 and $512 \mu \mathrm{g} / \mathrm{ml}$. The extract of $S$. birrea showed moderate activity against one of the two strains of C. albicans, as well as that of A. seyal against Streptoccocus mutans. These results highlight the interest of these three woody species in the context of oral hygiene as toothbrush sticks.

(C) 2019 International Formulae Group. All rights reserved.

Keywords: Chewing sticks, Antimicrobial activities, Peul, Tessékéré, North Ferlo, Senegal.

\section{INTRODUCTION}

La carie dentaire est une maladie multifactorielle résultant de la sensibilité de la paroi dentaire, associée à la présence de bactéries cariogènes, favorisée par un régime alimentaire riche en sucre notamment en sucrose (Galmiche, 2011). La carie dentaire serait apparue au Néolithique il y a environ 7000 ans avec la consommation de la farine obtenue par la culture des céréales (Pasquini, 2013). Cette période marque le passage de populations jusqu'alors nomades à un mode de vie sédentaire. Le même type de transition semble s'être établi au Ferlo depuis la création des forages dans une tentative de sédentarisation des populations autrefois nomades (Ancey et al., 2009), modifiant ainsi leur comportement alimentaire. Or, la consommation au Ferlo de produits d'addiction tels que l'alcool et le tabac au-delà d'un certain seuil, associée à la forte consommation de produits tels que le lait et le thé (produits alimentaires positivement corrélés au CPITN (Community Periodontal Index of Treatment Needs), un indice mesurant le besoin de traitement parodontal d'une communauté, sont à l'origine de la détérioration de la santé orale (Diouf et al., 2012). Pourtant, l'état bucco-dentaire joue un rôle important dans la santé humaine en générale. En effet, une santé bucco-dentaire dégradée conduit à une détérioration de la santé humaine avec des risques de diabète, d'hypertension et d'ostéoporose (Radoi et al., 2016). Heureusement, les caries dentaires et les maladies parodontales peuvent être évitées en contrôlant la formation de la plaque dentaire par l'action de la brosse dentaire ou par l'usage de bâtonnets frotte-dents (Khan et al., 2000). Les populations du Ferlo privilégient le recours aux bâtonnets frottedents pour leur hygiène dentaire (Diouf et al., 2012). Cet usage est d'ailleurs répandu sur une base ancienne parmi de nombreuses populations (Khan et al., 2000). Des groupes chimiques trouvés dans certaines plantes utilisées auraient des actions positives sur l'état de santé bucco-dentaire. Le développement des caries dentaires est en effet associé à la présence de bactéries Grampositives des genres Streptococcus, Lactobacillus et Actinomycetes (Arshad et al., 2017). L'évaluation de l'activité biologique de bâtonnets frotte-dents sur des souches cariogènes et parodontales pathogènes est alors nécessaire. C'est pourquoi, nous avons initié cette étude afin de participer à l'information des populations du Ferlo dans le choix des espèces comme bâtonnets frottedents. L'évaluation de l'activité d'extraits de plantes utilisées contre des souches microbiennes sera sans aucun doute d'un apport certain dans l'amélioration de la santé bucco-dentaire au niveau de la zone d'étude.

\section{MATERIEL ET METHODES \\ Zone d'étude}

Les enquêtes ont été menées dans la commune de Tessékéré (Ferlo Nord), zone où la dégradation de l'écosystème a conduit à plusieurs tentatives de restauration du couvert végétal. Le Ferlo couvre une superficie de $75000 \mathrm{~km}^{2}$ répartie entre les régions administratives nord du Sénégal (Saint Louis, 
Louga et Matam) (Figure 1). Il se localise entre la vallée du fleuve Sénégal (au Nord et à l'Est), le lac de Guiers (à l'Ouest) et la vallée fossile du Ferlo (au Sud) et comporte les caractéristiques climatiques des milieux sahéliens marqués par deux saisons: une saison des pluies de juillet à octobre assez variable depuis l'avènement des sécheresses des années 1970 avec des précipitations annuelles variant entre 300 et $600 \mathrm{~mm}$ et une saison sèche qui dure 8 à 9 mois, de novembre à juin (Diatta et al., 2019). La végétation est de type steppe avec une strate herbacée dominée par les graminées et des espèces ligneuses de petite taille (arbre et arbustes), en majorité épineuses et clairsemées (Ndong et al., 2015). D'un point de vue démographique, la population du Ferlo est constituée de Peuls, de Wolofs et de Maures (Niang et al., 2014).

\section{Enquêtes ethnobotaniques}

Les données ethnobotaniques ont été obtenues à l'aide d'un guide d'entretien semi directif administré de manière aléatoire aux populations enquêtées (Grenand et al., 2004). La méthodologie qualitative utilisée consiste à continuer l'entretien jusqu'à ce qu'il n'y ait plus de données nouvelles (saturation). Cette méthode nous a permis de recueillir différents usages des végétaux à visée cosmétique grâce à l'aide d'un guide-interprète maîtrisant à la fois la langue locale (Peul) et le Wolof (langue de traduction). Le guide est confectionné de manière à collecter les noms locaux des plantes utilisées, les parties utilisées et la partie du corps ciblée par l'usage. Le choix des personnes à interroger (personnes de plus de 15 ans) ciblant de façon prioritaire les femmes âgées, reconnues de façon générale comme détentrices de ces savoirs s'est fait avec l'aide de la population locale. Un interprète possédant une bonne connaissance de la langue locale a été associé aux entretiens et les noms des plantes ont été donnés par les enquêtés eux-mêmes en langue locale peule. Des sorties de terrain ont ensuite été effectuées pour identifier les espèces indiquées et prélever des échantillons pour la réalisation d'herbiers. Les conversations anodines et les observations directes ont également permis de recueillir les savoirs et savoir-faire sur le sujet. L'identification des espèces citées a été faite le plus souvent sur place à l'aide d'ouvrages de référence (Berhaut, 1967) et confirmée par comparaison avec des échantillons d'herbier de l'Herbier IFAN de l'Institut Fondamental d'Afrique Noire Cheikh Anta Diop de l'UCAD. Les noms d'espèces ont été validés sur la base de données CJB, Conservatoire et Jardin Botanique de la ville de Genève $<$ http://www.ville-

ge.ch/musinfo/bd/cjb/africa/>. Ne sont concernées par cette étude que les espèces utilisées comme bâtonnets frotte-dents pour l'hygiène bucco-dentaire.

La cohérence des informations est vérifiée selon la technique d'El Rhaffari et al., (2002). Ce dernier considère qu'une information est cohérente lorsqu'elle est rapportée au moins deux fois dans deux localités différentes par des informateurs différents, sinon elle est dite divergente. Seules les informations cohérentes ont été retenues dans le traitement des données. Ce traitement a été réalisé par des statistiques descriptives notamment le calcul de l'indice de fidélité (IF) selon la méthode de Begossi (1996) d'après la formule suivante :

$\mathrm{IF}=\mathrm{Ip} / \mathrm{Iu} \times 100$

Avec Ip le nombre d'informateurs ayant affirmé l'emploi d'une espèce précise dans une catégorie d'usage donnée et Iu le nombre total d'informateurs ayant cités la plante dans n'importe quelle catégorie d'usages. N'ont été sélectionnées que les plantes ayant été citées 5 fois au minimum et ayant un IF supérieur à 20 excepté pour $B$. senegalensis, espèce dont aucun informateur n'a mentionné une fonction curative.

\section{Extraction}

Afin de rester en cohérence avec l'utilisation traditionnelle des bâtonnets, les extraits ont été obtenus en milieu aqueux à pH 7 comme décrit par Rotimi et al. (1988) en adaptant un protocole en cours au laboratoire (Houël et al., 2017) pour l'étude des extractibles du bois. Les bâtonnets non débarrassés des écorces ont été réduits en poudre, puis 300 à $500 \mathrm{mg}$ de poudre ont été placés dans un tube en verre avec $6 \mathrm{ml}$ d'eau à $\mathrm{pH} 7$ et soumis à extraction aux ultrasons pendant une heure. Après centrifugation 
pendant 2 min et filtration (filtres de porosité $0,45 \mu \mathrm{m})$, l'eau a été évaporée. Une seule extraction a été réalisée pour chaque espèce étudiée.

Les solutions d'extraits utilisés pour les essais d'activité antimicrobienne ont été préparées en premier lieu dans le DMSO à $100 \mathrm{mg} / \mathrm{ml}$ puis diluées pour obtention d'une solution homogène à une concentration de 2 $\mathrm{mg} / \mathrm{ml}$ dans un milieu de culture aqueux compatible avec tout type de microorganismes (RPMI-1640 avec glutamine, sans bicarbonate et avec un indicateur au rouge de phénol).

\section{Activité antimicrobienne}

L'activité antimicrobienne des extraits aqueux de bâtonnets frotte-dents a été évaluée par des tests de microdilution.

\section{Activité antifongique}

Deux souches de référence de Candida albicans maintenues en collection au laboratoire ont été utilisées pour cet essai : $C$. albicans ATCC 10231 et C. albicans DSM 1386. Les levures ont été maintenues sur PDA (Potato Dextrose Agar) et mises en culture sur un milieu neuf à $32^{\circ} \mathrm{C} 48 \mathrm{~h}$ avant le test. Les essais d'activité antifongiques ont été réalisés selon un protocole déjà publié au laboratoire (Houël et al., 2014). Brièvement, des solutions standardisées de levures ont été préparées dans l'eau stérile en ajustant la turbidité à 0,5 selon le standard de McFarland de façon à obtenir $10^{5}$ à $10^{6}$ unités formant des colonies par millilitre de solution. Les suspensions ont ensuite été diluées au $1 / 1000^{\mathrm{e}}$ (v/v) dans le milieu de culture RPMI-1640. Les solutions d'extraits diluées dans le DMSO puis le RPMI comme indiqué ci-dessus ont été testées à des concentrations allant de 512 à $1 \mu \mathrm{g} / \mathrm{ml}$. Le kétoconazole a été utilisé comme témoin positif et testé à des concentrations allant de 64 à $0,125 \mu \mathrm{g} / \mathrm{ml}$. Les plaques ont été incubées pendant $48 \mathrm{~h}$ à $32^{\circ} \mathrm{C}$. Les valeurs de CIM (concentrations inhibitrices minimales) sont définies comme les concentrations les plus basses conduisant à une inhibition visible de la croissance fongique pour les extraits, et à celles inhibant $80 \%$ de la croissance fongique visible pour l'antifongique de référence. Tous les essais ont été répétés en duplicata sur chaque plaque.

\section{Activité antibactérienne}

Pour la réalisation des essais d'activité antibactérienne, 3 souches bactériennes de référence ont été obtenues auprès de l'Institut Pasteur à Paris: Streptococcus mutans (CIP103220T), Lactobacillus acidophilus (CIP76.13T) et Porphyromonas gingivalis (CIP103683T). Les souches ont été maintenues en milieu LB Lennox pour $S$. mutans (liquide, $37{ }^{\circ} \mathrm{C}$ ) et $L$. acidophilus (solide, $30^{\circ} \mathrm{C}$ ) et milieu solide Actinomycètes Vegiton (Fluka) à $37^{\circ} \mathrm{C}$ pour $P$. gingivalis. La méthode utilisée pour le test de microdilution a été adaptée de la littérature (Wiegand et al., 2008). Brièvement, les solutions de bactéries pour la réalisation du test ont été préparées par mise en suspension dans de l'eau stérile. Toutes les suspensions ont été ajustées visuellement au standard de turbidité McFarland 0,5 avant dilution au 1:100 (v/v) dans le milieu de culture adéquat (LB Lennox ou Actinomycètes Vegiton suivant la souche considérée). Les solutions d'extraits diluées dans le DMSO puis le RPMI comme indiqué ci-dessus ont été testées à des concentrations allant de 512 à $1 \mu \mathrm{g} / \mathrm{ml}$. Deux antibiotiques ont été choisis comme témoins positifs (le chloramphénicol et la streptomycine) et testés à des concentrations allant de 64 à 0,125 $\mu \mathrm{g} / \mathrm{ml}$. Les plaques ont été incubées à $37{ }^{\circ} \mathrm{C}$ pour $S$. mutans et $P$. gingivalis et à $30^{\circ} \mathrm{C}$ pour L. acidophilus. Les résultats ont été observés pour une croissance homogène et visible des microorganismes dans les puits de contrôle, soit après $24 \mathrm{~h}$ d'incubation pour $L$. acidophilus, $48 \mathrm{~h}$ pour $S$. mutans et $72 \mathrm{~h}$ pour $P$. gingivalis. En parallèle des contrôles visuels de la pureté des colonies ont été réalisés par mise en culture de la solution d'inoculum utilisée pour le test de microdilution sur le milieu solide adéquat. $\mathrm{La}$ CIM (concentration inhibitrice minimale) est déterminée soit comme la concentration correspondante au dernier puits où la croissance bactérienne est absente dans le cas des extraits, soit comme la concentration correspondante au dernier puits où la croissance bactérienne atteint au maximum $20 \%$ de la croissance bactérienne témoin dans le cas des antibiotiques de référence. Tous les essais ont été répétés en duplicata sur chaque plaque. 

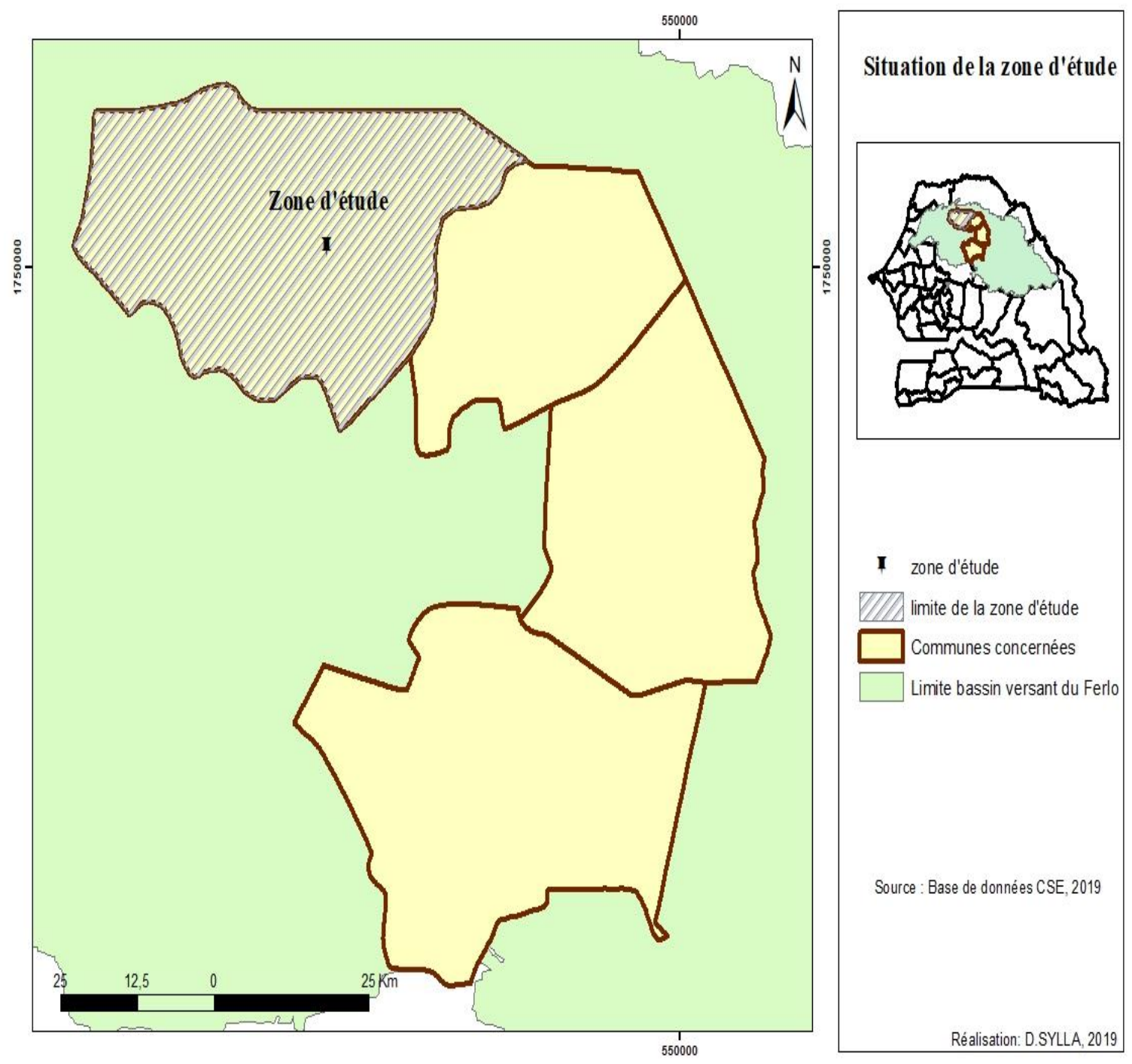

Figure 1 : Situation géographique du Ferlo (carte des localités visitées lors de l'enquête).

\section{RESULTATS}

Diversité des plantes utilisées comme bâtonnets frotte-dents

A l'issue des enquêtes de terrain, 24 espèces, réparties dans 21 genres et 12 familles botaniques, ont été identifiées comme bâtonnets frotte-dents par les cent vingt-cinq (125) personnes interrogées (Tableau 1). Pour l'évaluation des activités antimicrobiennes, des extraits aqueux ont été préparés à partir des tiges des treize (13) plantes ainsi réparties en se basant sur l'indice de fidélité et le nombre de citations dans la catégorie (Tableau 2) :

- six (6) espèces avec un indice de fidélité maximal (100\%)

- trois espèces avec un indice élevé (entre $90 \%-99 \%)$

- une espèce dont l'indice est moyen (58\%)

- et trois à faible indice (entre 35\% - 19\%).

La plupart de ces espèces sont des arbres (7) ou des arbustes (5). Elles se 
répartissent dans dix (10) genres et huit (8) familles (Tableau 1). La famille des Fabaceae comportant cinq (5) espèces regroupées dans deux (2) genres est la plus diversifiée. Elle renferme quatre (4) Fabaceae-Mimosoideae (Acacia senegal, Acacia nilotica subsp adstringens, Acacia seyal et Acacia tortilis subs raddiana) et une FabaceaeCaesalpinoideae (Bauhinia rufescens). Le genre Acacia comprenant quatre (4) espèces est le plus commun. Suit la famille des Combretaceae avec deux espèces (Anogeissus leiocarpa, Guiera senegalensis). Toutes les autres familles ne sont représentées que par une espèce. La tige est l'unique organe utilisé dans l'hygiène buccodentaire (HBD). L'espèce la plus citée par les utilisateurs est Balanites aegyptiaca (113 citations, IF 92), devant Acacia senegal (87 citations, IF 100), Commiphora africana (85 citations, IF 99) et Sclerocarya birrea (84 citations, IF 98), puis Salvadora persica (34 citations, IF 100). Les espèces A. senegal, S. persica, B. rufescens, $M$. crassifolia et $Z$. mauritiana dont le niveau de fidélité est égal à cent (100) sont exclusivement utilisées comme bâtonnet frotte dent.

\section{Facteurs socio-culturels influant sur la sélection des espèces par les utilisateurs}

Il a été noté lors des entretiens avec la population que $C$. africana (Baddi) est une espèce très appréciée, car elle procure une haleine agréable. Il a en plus été signalé par les utilisateurs que les bâtonnets frotte-dents issus de S. birrea (Eeri) et d'A. senegal (Patuki) conjureraient tout démenti lors d'une discussion, et que ceux de Ziziphus mauritiana (Diabi) sont réputés comme portebonheur. La grande utilisation des bâtonnets de S. persica (Guddi) comme cure-dent par la population de la commune serait due à une raison religieuse. Il a par ailleurs été mentionné d'autres motivations de choix et/ ou de non choix de certains bâtonnets frottedents associées à des croyances et/ou de représentations. Effectivement, B. aegyptiaca et $C$. africana, espèces très résistantes à la sécheresse sont pour cette raison associées à la santé et à la longévité d'où leur usage. En revanche, l'usage de B. aegyptiaca et d'A. senegal comme bâtonnet frotte dent seraient déconseillés dans certains cas car, le premier stimulerait la montée du venin en cas de morsure de serpent et le second serait connu pour favoriser les conflits et disputes dans les maisons.

\section{Mesure de l'activité antimicrobienne}

Les résultats obtenus pour la mesure de l'activité antimicrobienne des extraits aqueux de bâtonnets frotte-dents sont présentés dans le Tableau 2. Les rendements d'extraction obtenus pour chaque espèce sont également précisés. Le rendement moyen obtenu est de 2,0 \% (déviation standard: 1,3 ), avec une valeur minimale de $0,8 \%$ pour $A$. seyal et une valeur maximale de 5,7 \% dans le cas de $M$. crassifolia. Parmi les espèces dont l'activité antimicrobienne a été évaluées, seule $A$. leiocarpa (Combretaceae) a présenté une activité antimicrobienne mesurable $(\leq 512$ $\mu \mathrm{g} / \mathrm{ml}$ ) vis-à-vis de 4 des 5 souches testées. Son activité est significative selon les critères proposés par Gertsch et al. (2009) vis-à-vis de C. albicans (CIM $16 \mu \mathrm{g} / \mathrm{ml}$ ) et $P$. gingivalis (CIM $128 \mu \mathrm{g} / \mathrm{ml}$ ). Deux autres espèces, $S$. birrea (Anacardiaceae) et A. seyal (Fabaceae) ont montré une activité modérée vis-à-vis d'une des souches testées (CIM de $512 \mu \mathrm{g} / \mathrm{ml}$ vis-à-vis de $C$. albicans et $S$. mutans respectivement). 
Tableau 1 : Liste des espèces recensées comme bâtonnets frotte-dents dans la commune Tessékéré, Ferlo Nord, Sénégal.

\begin{tabular}{|c|c|c|c|c|c|c|}
\hline Nom Peulh & Espèce & Famille & Organe & Ip & $\mathbf{I u}$ & IF $(\%)$ \\
\hline Gawdi & $\begin{array}{l}\text { Acacia nilotica subsp adstringens } \\
\text { (Schumach. \& Thonn.) Roberty }\end{array}$ & Fabaceae & tige & 21 & 36 & $58,33^{*}$ \\
\hline Thili & Acacia tortilis subsp raddiana Savi. & Fabaceae & tige & 29 & 119 & $24,36^{*}$ \\
\hline Patuki & Acacia senegal (L.) Willd. & Fabaceae & tige & 87 & 87 & $100^{*}$ \\
\hline Bulbi & Acacia seyal Del & Fabaceae & tige & 16 & 84 & $19,04^{*}$ \\
\hline kodioli & $\begin{array}{l}\text { Anogeissus leiocarpus (DC.) Guill. \& } \\
\text { Perr }\end{array}$ & Combretaceae & tige & 5 & 14 & $35,71^{*}$ \\
\hline Muthiteki & Balanites aegyptiaca $($ L.) Delile & Zygophyllaceae & tige & 113 & 123 & $91,86^{*}$ \\
\hline Namari & Bauhinia rufescens Lam. & Fabaceae & tige & 15 & 15 & $100^{*}$ \\
\hline Gissili & $\begin{array}{l}\text { Boscia senegalensis (Pers.) Lam. ex } \\
\text { Poir. }\end{array}$ & Capparaceae & tige & 8 & 8 & 100 \\
\hline Seing seing & Cadaba farinosa Forsk. & Capparaceae & tige & 1 & 1 & 100 \\
\hline Burli & $\begin{array}{l}\text { Dichrostachys cinerea (L) Wight \& } \\
\text { Arn }\end{array}$ & Fabaceae & tige & 1 & 1 & 100 \\
\hline Bakagni & Cissus populnea Guill. et Perr. & Vitaceae & tige & 1 & 1 & 100 \\
\hline Lawgnandi & Combretum aculeatum Vent & Combretaceae & tige & 1 & 1 & 100 \\
\hline Baddi & Commiphora africana (A.Rich.) Engl. & Burseraceae & tige & 85 & 86 & $98,83^{*}$ \\
\hline Dialambani & Dalbergia melanoxilon Guill. \& Perr. & Fabaceae & tige & 1 & 1 & 100 \\
\hline Kelli & Grewia bicolor Juss. & Malvaceae & tige & 3 & 64 & 4,68 \\
\hline Géloki & Guiera senegalensis J. F. Gmel. & Combretaceae & tige & 14 & 14 & $100^{*}$ \\
\hline Gial goti & $\begin{array}{l}\text { Gymnosperia senegalensis (Lam.) } \\
\text { Exell. }\end{array}$ & Celastraceae & tige & 1 & 1 & 100 \\
\hline Degeti & Maerua crassifolia Forks. & Capparaceae & tige & 13 & 13 & $100^{*}$ \\
\hline Koyli & Mitragyna inermis Willd & Rubiaceae & tige & 4 & 12 & 33,33 \\
\hline Guddi & Salvadora persica $\mathrm{L}$. & Salvadoraceae & tige & 34 & 34 & $100^{*}$ \\
\hline Eeri & Sclerocarya birrea (A Rich.) Hochst & Anacardiaceae & tige & 84 & 86 & $97,67^{*}$ \\
\hline Diadmi & Tamarindus indica $\mathrm{L}$. & Fabaceae & tige & 2 & 2 & 100 \\
\hline Kafaki & Waltheria indica $\mathrm{L}$. & Malvaceae & tige & 1 & 1 & 100 \\
\hline Diabi & Ziziphus mauritiana Lam. & Rhamnaceae & tige & 13 & 13 & $100^{*}$ \\
\hline
\end{tabular}

Ip: nombre d'informateurs ayant affirmé l'emploi de l'espèce dans l'Hygiène Bucco-Dentaire (HBD) $\boldsymbol{I} \boldsymbol{u}$ : nombre total d'informateurs ayant cités la plante dans n'importe quelle autre catégorie d'usage

*Espèces retenues pour l'évaluation de l'activité antimicrobienne 
Tableau 2: Rendement d'extraction et activité antimicrobienne des 13 espèces principales bâtonnets frotte-dents de la commune de Tessékéré, Ferlo Nord Sénégal.

\begin{tabular}{|c|c|c|c|c|c|c|}
\hline \multirow[t]{2}{*}{ Espèce } & \multirow{2}{*}{$\begin{array}{l}\text { Rendement } \\
\text { d'extraction }(\%)\end{array}$} & \multirow{2}{*}{$\begin{array}{l}\text { CIM } \quad \text { Candida } \\
\text { albicans } \\
\text { ATTC } 10231(\mu \mathrm{g} / \mathrm{ml})\end{array}$} & \multirow{2}{*}{$\begin{array}{lr}\text { CIM } & C . \\
\text { albicans } & \text { DSM } \\
1386(\mu \mathrm{g} / \mathrm{ml}) & \end{array}$} & Streptococcus & CIM Lactocillus & CIM Porphyromonas \\
\hline & & & & $\begin{array}{l}\text { mutans CIP 103220T } \\
(\mu \mathrm{g} / \mathrm{ml})\end{array}$ & $\begin{array}{ll}\text { acidophilus } & \text { CIP } \\
\text { 76.13T }(\mu \mathrm{g} / \mathrm{ml}) & \end{array}$ & $\begin{array}{l}\text { gingivalis CIP } \\
103683 \mathrm{~T}(\mu \mathrm{g} / \mathrm{ml})\end{array}$ \\
\hline Balanites aegyptiaca & 1,8 & - & - & - & - & - \\
\hline Acacia senegal & 3,0 & - & - & - & - & - \\
\hline Commiphora africana & 1,7 & - & - & - & - & - \\
\hline Sclerocarya birrea & 1,2 & - & 512 & - & - & - \\
\hline $\begin{array}{l}\text { Acacia tortilis subsp } \\
\text { raddiana }\end{array}$ & 1,0 & - & - & - & - & - \\
\hline Vachellia nilotica & 2,9 & - & - & - & - & - \\
\hline Salvadora persica & 3,1 & - & - & - & - & - \\
\hline Guiera senegalensis & 1,0 & - & - & - & - & - \\
\hline Bauhinia rufescens & 1,8 & - & - & - & - & - \\
\hline Acacia seyal & 0,8 & - & - & 512 & - & n.t. \\
\hline Ziziphus mauritiana & 1,1 & - & - & - & - & - \\
\hline Maerua crassifolia & 5,7 & - & - & - & - & - \\
\hline Anogeissus leiocarpa & 1,5 & 1,5 & 512 & 16 & 512 & 128 \\
\hline Cétoconazole & & & & n.t. & n.t. & n.t. \\
\hline Streptomycine & & & & $<0,125$ & $<0,125$ & 16 \\
\hline Chloramphénicol & & & & 1 & 0,5 & 0,5 \\
\hline
\end{tabular}

- : extrait non actif $(\mathrm{CIM}>512 \mu \mathrm{g} / \mathrm{ml}) ; \quad$ n.t: non testé

En gras: espèces présentant une activité antimicrobienne mesurable (>512 $\mu \mathrm{g} / \mathrm{ml})$ sur un ou plusieurs des micro-organismes testés. 


\section{DISCUSSION}

La tige est le seul organe de la plante utilisé comme bâtonnet frotte-dent (Tableau 1) tandis que la racine et la feuille l'emportent dans le traitement de la constipation chez les Malinké de Tomboronkoto (Guèye, 2012). Les motifs de choix convoqués dans l'élection des bâtonnets frotte-dents sont multiples et variés. Ils vont de la disponibilité ( $B$. aegyptiaca, A. senegal, A. nilotica) de la plante aux croyances populaires associées à certaines espèces en passant par les propriétés médicinales ( $S$. persica et $A$. nilotica), la tendresse ou non des fibres de la plantes $(B$. aegyptiaca et $S$. persica), l'odeur agréable ( $C$. africana) ou non de la plante, le goût amer ( $B$. aegyptiaca et $A$. nilotica) ou non (A. senegal) de la plante, la forme de la tige de la plante $(S$. persica et $B$. aegyptiaca). En plus, les fonctions du bâtonnet frotte-dent (action mécanique consistant au nettoyage des surfaces, massage gingival favorisant la circulation sanguine, asepsie orale à fonction phytothérapie et la stimulation des structures parodontales) sont multiples et variés (Akpona et al., 2009). Le même auteur a même établi une classification des bâtonnets frotte-dents utilisés basée sur la perception locale. Dans cette même perspective, Shepard (2004) recommande des investigations sur les propriétés organoleptiques des espèces.

Par ailleurs, des facteurs culturels peuvent se révéler très influents sur le choix des plantes par les utilisateurs (MenendezBaceta et al., 2015). Les croyances et représentations sociales mentionnées au Ferlo renvoient plus souvent à la chance $(Z$. mauritiana), à la volonté de garder le monopole d'une conversation ( $S$. birrea), à la santé (B. aegyptiaca et $C$. africana), à la religion (S. persica, B. rufescens, C. africana et $A$. nilotica), à la longévité (B. aegyptiaca et $C$. africana) et à la quête d'une richesse ( $Z$. mauritiana). L'usage des plantes utilisées pour des raisons religieuses est dopé par le fait que ces plantes furent les cure-dents favoris du Prophète Mahomet PSL tandis que la forme incurvée des épines d'A. senegal attesterait qu'une telle plante soit génératrice de dispute et de conflits.
Certaines espèces comme $B$. aegyptiaca, A. senegal, A. tortilis et $A$. nilotica, plantées dans le cadre du programme de la Grande Muraille Verte sont appréciées des populations. Elles sont prisées pour divers aspects : alimentaires (fruit), énergétique (bois de chauffage) et médicinal (Niang et al., 2014).

Les rendements d'extraction obtenus sont notablement différents en fonction des espèces. Cependant, ces mesures étant des valeurs brutes (rendements totaux) et non des valeurs de rendement d'extraction de classes données de composés chimiques, elles ne peuvent être corrélées aux valeurs d'activité biologiques mesurées, celles-ci ne dépendant que de la nature des molécules présentes comme illustré par exemple dans les travaux de Basset et al. (2012).

Seuls les extraits d'A. leiocarpa ont montré une activité antimicrobienne significative. Pour cette espèce le rendement d'extraction était de $1,5 \%$ pour une moyenne de 2,0\%. Ces résultats corroborent différentes études sur cette espèce. En effet, une valeur de CIM de $500 \mu \mathrm{g} / \mathrm{ml}$ avait été obtenue pour un extrait éthanolique d'écorce de tige vis-à-vis de C. albicans (Kubmarawa et al., 2007). Les extraits méthanoliques $70 \%$ et aqueux des écorces de tige de A. leiocarpa ont eu un effet bactéricide sur $100 \%$ des souches bactériennes testées par Sanogo et al. (2016). Diatta et al. (2019) ont montré que l'extrait aqueux de bâtonnets frotte-dents de tiges de $S$. birrea est d'une richesse phytochimique intéressante avec des composés de classes variées (stéroïdes, tanins, saponines, anthocyanines, coumarines, émodines). De même, cette espèce s'est révélée comme l'une des plus riches en polyphénols (348 $\mu \mathrm{g} / \mathrm{ml})$, flavonoïdes (303 $\mu \mathrm{gEQ} / \mathrm{mg}$ ) et alcaloïdes (46 $\mu \mathrm{g} / \mathrm{mg}$ ) (Diatta et al., 2019). Ces résultats confirmeraient l'activité antimicrobienne observée qui serait liée à la présence de métabolites secondaires bioactifs comme noté par Akpona et al. (2009).

D'autres équipes ayant travaillé sur $S$. birrea, ont mesuré une activité anticandidale faible pour des extraits éthanolique et aqueux d'écorce de tige avec une concentration 
inhibitrice minimale de $1 \mathrm{mg} / \mathrm{ml}$ (Nciki et al., 2016) et une activité modérée pour un extrait méthanolique de racines (CIM $250 \mu \mathrm{g} / \mathrm{ml}$ ) (Hamza et al., 2006). Par ailleurs, l'activité antibactérienne d'extraits divers (acétone, méthanol, hexane, eau) d'écorce de tige étudiée sur des souches variées a révélé une faible activité (CIM > $1 \mathrm{mg} / \mathrm{ml}$ ) à modérée (CIM de 490-810 $\mu \mathrm{g} / \mathrm{ml}$ ) suivant les cas (Nndwammbi et al., 2018). Une valeur de CIM de $625 \mu \mathrm{g} / \mathrm{ml}$ a été mesurée sur une souche de Streptococcus (S. pyogenes) pour un extrait aqueux d'écorce de tige (Tanih et al., 2012). D'ailleurs, au Sénégal, notamment dans la communauté de Tomboronkoto, les écorces de $S$. birrea sont recommandées dans le traitement de l'odontalgie (Guèye, 2012).

Acacia seyal a montré une activité modérée (CIM de $512 \mu \mathrm{g} / \mathrm{ml}$ ) vis-à-vis de la souche de S. mutans. Mohieldin et al. (2017) dévoilent une faible activité antimicrobienne mais mesurable des écorces et de bois d'A. seyal sur: Streptococcus sobrinus, bactérie identifiées comme un des pathogènes majeurs de la cavité bucco-dentaire et sur $P$. gingivalis, bactérie fortement impliquée dans l'étiologie des parodontites. Des activités antibactériennes notables ont été mesurées par ailleurs, avec des CIM allant de moins de 0,1 $\mathrm{mg} / \mathrm{ml}$ à $0,4 \mathrm{mg} / \mathrm{ml}$ pour des extraits éthanoliques d'écorce vis-à-vis de Bacillus subtilis, Staphylococcus aureus et Klebsiella pneumoniae (Eldeen et Van Staden, 2007).

D'après les études de Kubmarawa et al. (2007) et Mohieldin et al. (2017a), les extraits d'écorce et de racines de $B$. aegyptiaca, l'espèce la plus citée dans notre enquête n'ont aucune activité vis-à-vis de $C$. albicans et Streptococcus sobrinus (CIM $\geq 4 \mathrm{mg} / \mathrm{ml}$ ) confirmant ainsi nos résultats. Toutefois, Diouf et al. (2011) rapporte un intérêt de son utilisation comme cure-dents contre les caries au Sénégal. En plus, B. aegyptiaca est réputé anti-caries au Soudan (Mohieldin et al., 2017a). Eldeen et Van Staden (2007) avec des extraits au méthanol et à l'acétate d'éthyle d'écorce de B. aegyptiaca ont obtenu une activité antibactérienne notable vis-à-vis d'un panel de souches, ce qui ne fut pas le cas avec des extraits éthanoliques, montrant ainsi l'importance du solvant utilisé. De nombreuses raisons (parties de la plante, solvant d'extraction, biotope de la plante et éventuellement le moment de la collecte) pourraient effectivement expliquer les différences d'activité entre les études.

Nous n'avons noté aucune valeur notable d'activité antimicrobienne pour l'extrait de $S$. persica, espèce pourtant très connue pour son usage en tant que bâtonnet frotte-dent (Garba et al., 2017 ; Aumeeruddy et al., 2018). Ceci n'est pourtant pas en contradiction avec des résultats de la littérature. Avec respectivement des CIM d'écorce de la tige de $1 \mathrm{mg} / \mathrm{ml}$ pour les extraits méthanoliques et $4 \mathrm{mg} / \mathrm{ml}$ pour les extraits hydroéthanolique vis-à-vis de $P$. gingivalis et aucune valeur mesurée pour les extraits de bois (Mohieldin et al., 2017b). Parmi les nombreux résultats d'essais d'activité antimicrobienne figurent nombre de solvants d'extraction, protocoles d'essais et de micro-organismes différents, rendant difficile la comparaison des diverses données obtenues et pouvant en même temps expliquer les différences de conclusions tirées (Hadacek et Greger, 2000).

Aucune corrélation n'est notée entre l'activité biologique mesurée dans nos essais et l'indice de fidélité d'une part, et les critères socio-culturels influençant le choix des espèces comme bâtonnets frotte-dents d'autre part. Les extraits les plus actifs ne sont pas ceux des espèces les plus citées.

\section{Conclusion}

La population de la commune de Tessékéré exploite 24 plantes comme curedents dont les plus populaires (13) ont fait l'objet d'une évaluation de leurs activités antimicrobiennes. Toutes les espèces sont potentiellement bien adaptées à la zone d'étude et pourraient dès lors être incluses dans les programmes de reforestation de la Grande Muraille Verte. L'utilisation des espèces est fortement influencée par divers facteurs socio-culturels allant de la perception sociale à la religion. Les résultats obtenus dans le cadre de cette étude ont permis, au vu des activités antimicrobiennes mesurées, de 
mettre en évidence l'intérêt de l'utilisation de A. leiocarpa comme bâtonnet frotte-dents. L'usage de A. seyal et de $S$. birrea s'est aussi révélé bénéfique dans l'hygiène buccodentaire. Même si aucune activité antimicrobienne n'a été notée chez les dix autres espèces testées, il n'en demeure pas moins que leur utilisation comme bâtonnets frotte-dents puisse contribuer à une meilleure prise en compte de l'hygiène bucco-dentaire chez la population du Ferlo par action mécanique en éliminant la plaque dentaire. Ainsi, il nous paraît nécessaire de poursuivre l'étude et de l'étendre à d'autres sites pour une amélioration de l'état sanitaire des populations rurales.

\section{CONFLIT D'INTERETS}

Les auteurs déclarent qu'ils n'ont pas de conflit d'intérêts.

\section{CONTRIBUTIONS DES AUTEURS}

BDD: L'article s'inscrit dans le cadre de ma thèse sur les pratiques cosmétiques traditionnelles. Ainsi, j'ai mené les enquêtes de terrain, le dépouillement et le traitement des données et initié la rédaction de cet article. EH a réalisé les extractions, a évaluée l'activité antimicrobienne des extraits et a également largement contribué à la rédaction du document. MG est Botaniste ethnobotaniste et m'a initié à l'ethnobotanique, a été avec moi sur le terrain pendant les enquêtes. Il a aussi grandement contribué au traitement des données et à la rédaction de cet article. $\mathrm{ON}$ est chimiste et a participé à l'analyse des données et à la rédaction du document. GB est socioanthropologue spécialiste du corps et a participé à l'analyse des données d'enquête notamment sur le lien entre usage des plantes, représentations socioculturelles associées aux plantes.

\section{REMERCIEMENTS}

Nous remercions particulièrement le labex Drihm qui, à travers l'Observatoire Homme Milieu de Tessekéré a financé ce projet, facilitant ainsi énormément le travail. Les auteurs remercient également chaleureusement les populations de la commune de Tessékéré pour avoir accepté de partager leurs connaissances dans le cadre de cette étude.

\section{RÉFÉRENCES}

Akpona HA, Akpona JDT, Awokou SK, Yemoa A, Dossa LOSN. 2009. Inventory, folk classification and pharmacological properties of plant species used as chewing sticks on Benin Republic. Journal of Medicinal Plants Research, 3: 382-389. DOI: 10.5897/JMPR

Ancey V, Ickowicz A, Corniaux C, Manoli C, Magnani S. 2009. Stratégies pastorales de sécurisation chez les Peuls du Ferlo (Sénégal). Journal des Africanistes, 78(1-2) : $\quad$ 105-119. DOI : http://journal.openedition.org/africaniste/ 2280

Arshad MS, Hussain I, Mahmood MS, Khan MN. 2017. Evaluation of antimicrobial potential of Acacia nilotica (Kikar) against oral pathogens associated with caries and periodontitis. Pakistan Journal of Agricultural Sciences, 54: 423-430.

DOI: 10.21162/PAKJAS/17.6385

Aumeeruddy MZ, Zengin G, Mahomoodally MF. 2018. A review of the traditional and modern uses of Salvadora persica L. (Miswak): Toothbrush tree of Prophet Muhammad. Journal of Ethnopharmacology, 213: 409-444. DOI: 10.1016/j.jep.2017.11.030

Basset C, Rodrigues AMS, Eparvier V, Silva MRR, Lopes NP, Sabatier D, Fonty E, Espindola LS, Stien D. 2012. Secondary metabolites from Spirotropis longifolia (DC) Baill and their antifungal activity against human pathogenic fungi. Phytochemistry, 74: 166-172. DOI: 10.1016/j.phytochem.2011.10.011

Begossi A.1996. Use of ecological methods in ethnobotany: Diversity Indices. 
Economic Botany, 50: 280. DOI: 10.1007/10-1007/36-02907333

Berhaut J. 1967. Flore du Sénégal (2nd éd). Clairafrique : Dakar.

Diatta BD, Niass O, Diouf $\mathrm{M}$, Guèye $\mathrm{M}$, Houel E, Boetsch G. 2019. Diversité et composition phytochimique des plantes utilisées comme bâtonnets frotte-dents (cure-dents) par les Peul de la commune de Tessekere (Ferlo Nord Sénégal). Phytothérapie Recherche. DOI : 10.3166/phyto-2019-0161

Diouf M, Boetsch G, Cissé D, Tal-Dia A, Bonfil JJ. 2012. Mode de vie et santé bucco-dentaire chez les populations peules du Ferlo au Sénégal. Médecine et Santé Tropicale, 22: 187-192. DOI: 10.1684/mst.2012.0053

Eldeen IMS, Van Staden J. 2007. In vitro pharmacological investigation of extracts from some trees used in Sudanese traditional medicine. South African Journal of Botany, 73: 435-440. DOI: 10.1016/j.sajb.2007.03.009

El Rhaffari L, Zaid A. 2002. Phytotherapy practice in south-eastern Morocco (Tafilalet). Empirical knowledge for a renovated pharmacopoeia. In From Knowledge Sources to Future Medicines, Fleury J. (ed.). Edition IRD: Paris; 293318.

Galmiche F. 2011. Le rôle de l'alimentation dans la santé bucco-dentaire. Thèse Chirugie Dentaire à la Faculté d'Odontologie, Univesité Henry Poincare- Nancy 1, 165 pages.

Garba ABH, Agarya M, Traore A. 2017. Etude des effets vermicide et antidiarrhéique du macéré aqueux des feuilles de Salvadora persica, L. (Salvadoraceae). Int. J. Biol. Chem. Sci., 11(1): 54-66. DOI: 10.4314/ ijbcs.v11i1.5

Gertsch J. 2009. How scientific is the science in ethnopharmacology? Historical perspectives and epistemological problems. Journal of Ethnopharmacology, 122: 177-183. DOI: 10.1016/j.jep.2009.01.010.

Grenand P, Moretti C, Jacquemin H, Prévost MF. 2004. Pharmacopées Traditionnelles en Guyane (1Ed.). IRD : Paris, France ; 816p.

http://www.documentation.ird.fr/hor/fdi: 010034335

Guèye M. 2012. Contribution à l'étude ethnobotanique chez les Malinké de la communauté rurale de Tomboronkoto (région de Kédougou) et valorisation des collections historiques de l'Herbier de l'Institut fondamental d'Afrique noire Cheikh Anta Diop (IFAN Ch. A. Diop) / UCAD. Thèse doctorat d'Etat, FST, UCAD, p.18 et p.142.

Guèye M, Cisse A, Diatta CD, Diop S, Koma S. 2012. Etude ethnobotanique des plantes utilisées contre la constipation chez les Malinké de la communauté rurale de Tomboronkoto, Kédougou (Sénégal). Int. J. Biol. Chem. Sci., 6 (2): 778-779.

DOI: http://dx.doi.org/10.4314/ijbcs.v6i2.19

Hadacek F, Greger H. 2000. Testing of antifungal natural products: methodologies, comparability of results and assay choice. Phytochemical Analysis, 11: 137-147. DOI: 10.1002/(sici)1099-

1565(200005/06)11:3<137::AIDPCA514>3.0.CO;2-I

Hamza OJM, Van Den Bout Van Den Beukel CJP, Matee MIN. 2006. Antifungal activity of some Tanzanian plants used traditionally for the treatment of fungal infections. J. Ethnopharmacol., 108 : 124-132.

DOI: 10.1016/j.jep.2006.04.026

Houel E, Rodrigues AMS, Jahn-Oyac A, Bassière J-M, Eparvier V, Deharo E, Stien D. 2014. In vitro antidermatophytic activity of Otacanthus azureus (Linden) Ronse essential oil alone and in 
combination with azoles. Journal of Applied Microbiology, 116: 288-294. DOI: 10.1111 jam.12377

Houël E, Rodrigues A, Nicolini E, Ngwete O, Duplais C, Stien D, Amusant N. 2017. Natural durability of Sextonia rubra, an Amazonian tree species: description and origin. IRG/WP 17-10887, Proceedings IRG Annual Meeting.

Khan MNNO, Olipa N, Mecky IN. 2000. Antimicrobial activity or Tanzanian chewing sticks against oral pathogenic microbes. Pharmaceutical Biology, 38(3): 235-240. DOI: $10.1076 / 1388$ 0209 (2000007)3831SFT235

Kubmarawa D, Ajoku GA, Enwerem NM, Okorie DA. 2007. Preliminary phytochemical and antimicrobial screening of 50 medicinal plants from Nigeria. African Journal of Biotechnology, 6: 1690-1696. DOI: 10.5897.AJB 2007.000-2246

Menendez-Baceta G, Aceituno-Mata L, Reyes-García V, Tardío J, Salpeteur M, Pardo-de-Santayana M. 2015. The importance of cultural factors in the distribution of medicinal plant knowledge: A case study in four Basque regions. Journal of Ethnopharmacology, 161: 116-127. DOI: 10.1016/j.jep.2014.12.007

Mohieldin EAM, Muddathir AM, Yamauchi K, Mitsunaga T. 2017a Anti-caries activity of selected Sudanese medicinal plants with emphasis on Terminalia laxiflora. Revista Brasileira de Farmacognosia, 27: 611-618. DOI: 10.1016/j.jbjp.2017.04.002

Mohieldin EAM, Muddathir AM, Mitsunaga T. 2017b. Inhibitory activities of selected Sudanese medicinal plants on Porphyromonas gingivalis and matrix metalloproteinase-9 and isolation of bioactive compounds from Combretum hartmannianum (Schweinf) bark. BMC Complementary and Alternative
Medicine, 17: 224 DOI: 10.1186/s12906-017-1735-y

Ndong AT, Ndiaye O, Sagna MB, Diallo A, Galop D, Guissé A. 2015. Caractérisation de la végétation ligneuse sahélienne du Sénégal : cas du Ferlo. International Journal of Biological and Chemical Sciences, 9: 2582-2594. DOI: 10.4314/ijbcs.v9i6.6

Nciki S, Vuuren S, van Eyk A, de Wet $\mathrm{H}$. 2016. Plants used to treat skin diseases in northern Maputaland, South Africa: antimicrobial activity and in vitro permeability studies. Pharmaceutical Biology, 54: 2420-2436. DOI: 10.3109/13880209.2016.1158287

Niang K, Sagna MB, Ndiaye O, Thiaw A, Diallo A, Akpo LE, Saleh MM, Diome N, Diatta S, Faye MN, Gueye M, Guissé A, Goffner D. 2014b. Revisiting tree species availability and usage in the Ferlo region of Senegal: a rationale for indigenous tree planting strategies in the context of the Great Green Wall for the Sahara and the Sahel initiative. Journal of Experimental Biology and Agricultural Sciences, 2: 529-537.

Nndwammbi M, Ligavha-Mbelengwa MH, Anokwuru CP, Ramaite IDI. 2018. The effects of seasonal debarking on physical structure, polyphenolic content and antibacterial and antioxidant activities of Sclerocarya birrea in the Nylsvley nature reserve. South African Journal of Botany, 118: 138-143. DOI: 10.1016/j.jsab.2018.06.018

Pasquini A. 2013. Pour Offrir un Sourire Evolution de L'hygiène Bucco-Dentaire au Fil des Siècles et des Civilisations. Editions universitaires européennes ; $340 \mathrm{p}$.

Radoi L, Veille Finet A, Dupuis V, Folliguet M .2016. Impact de l'état bucco-dentaire sur la santé générale: actualisation des connaissances : review of the impact of 
oral diseases on general health. Rev Geriatr., 41 (1): 5-16.

Rotimi VO, Laughon BE, Bartlett JG, Mosadomi HA. 1988. Activities of Nigerian chewing sticks extracts against Bacteroides gingivalis and Bacteroides melaninogenicus. Antimicrobial Agents and Chemotherapy, 3: 598-600. DOI: 10.1128/aac.32.4.598

Sanogo Y, Guessennd NK, Tra Bi HF, Kouadio NJ, Konan FK, Bamba M, Danho N, Bakayoko A, Yao K, Dosso M . 2016. Evaluation in vitro de l'activité des écorces de tige de Anogeissus leiocarpus (DC) Guill. et Perr. (Combretaceae) sur des bactéries responsables de maladies courantes en Afrique et criblage phytochimique. Int. J. Biol. Chem. Sci., 10(3): 1139-1152. DOI: $10.4314 /$ ijbcs.v10i3.19
Shepard G. 2004. A sensory ecology of medicinal plant therapy in two Amazonian societies. American Anthropologist, 106: 252-266. DOI: 10.1525/aa.2004.106.2.252

Tanih NF, Ndip RN. 2012. Evaluation of the acetone and aqueous extracts of mature stem bark of Sclerocarya birrea for antioxidant and antimicrobial properties. Evidence-Based Complementary and Alternative Medicine, 2012: 834156. DOI: $10.1155 / 2012 / 834156$

Wiegand I, Hilpert K, Hancock EW. 2008. Agar and broth dilution methods to determine the minimal inhibitory concentration (MIC) of antimicrobial substances. Nature Protocols, 3: 163175. DOI: $10.1038 /$ nprot.2007.521 